\title{
Intoxicação espontânea por Senna obtusifolia em bovinos no Pantanal Sul-Mato-Grossense ${ }^{1}$
}

\begin{abstract}
Amanda Q. de Carvalho², Nilton M. Carvalho ${ }^{3}$, Gustavo P. Vieira ${ }^{4}$, Ariany C. dos Santos ${ }^{2}$, Gumercindo L. Franco ${ }^{3}$, Arnildo Pott ${ }^{5}$, Cláudio S.L. Barros ${ }^{6}$ e Ricardo A.A. Lemos ${ }^{3 *}$

ABSTRACT.- Carvalho A.Q., Carvalho N.M., Vieira G.P., Santos A.C., Franco G.L., Pott A., Barros C.S.L. \& Lemos R.A.A. 2014. [Spontaneous poisoning by Senna obtusifolia in cattle of the southern Pantanal.] Intoxicação espontânea por Senna obtusifolia em bovinos no Pantanal Sul-Mato-Grossense. Pesquisa Veterinária Brasileira 34(2):147-152. Faculdade de Medicina Veterinária e Zootecnia, Universidadade Federal de Mato Grosso do Sul, Av. Senador Filinto Müller 2443, Campo Grande, MS 79074-460, Brazil. E-mail: ricardo.lemos@ufms.br

Senna sp. poisoning in livestock has been reported in several occasions in Brazil usually from southern Brazil and involving S. occidentalis as the culprit. The objective of this study is to report the occurrence of an outbreak of $S$. obtusifolia poisoning in cattle in the Pantanal Region of Mato Grosso do Sul, Brazil. In a herd of 313 heifers, 165 were affected and died (morbidity rate of $52.7 \%$ and lethality rate of $100 \%$ ). The 313 heifers remained in the paddock infested by S. obtusifolia for 37 days. Clinical signs consisted of reluctance to move, incoordination, permanent sternal recumbency, decrease in the tonus of the tongue, alertness, myoglobinuria characterized by dark brown urine, and dry stools with or without mucus; or occasionally diarrhea with streaks of blood. The main gross findings in 12 necropsied cattle were in the skeletal muscles of the hind limbs, and were characterized by varying degrees of paleness of muscle groups. Histologically, the most relevant lesion was segmental multifocal degeneration and necrosis in striated skeletal muscles (multifocal lypolyphasic toxic degenerative myopathy). The epidemiological, clinical and pathological data allowed to conclude for the diagnosis of poisoning by S. obtusifolia in this outbreak.
\end{abstract}

INDEX TERMS: Poisonous plants, cattle diseases, Senna obtusifolia, Fabaceae, Caesalpinioideae, coffee senna, plant poisoning, Centro-Oeste, disease of striated muscle.

RESUMO.- Descreve-se um surto de intoxicação por Senna obtusifolia em bovinos no estado de Mato Grosso do Sul, na região do Pantanal. Em um lote de 313 novilhas, 165 adoeceram e morreram (coeficientes de morbidade de 52,7\% e de letalidade de 100\%). Os bovinos permaneceram no

\footnotetext{
${ }^{1}$ Recebido em 21 de janeiro de 2014.

Aceito para publicação em 6 de fevereiro de 2014.

${ }^{2}$ Programa de Pós-Graduação em Ciência Animal, Faculdade de Medicina Veterinária e Zootecnia (FAMEZ), Universidade Federal de Mato Grosso do Sul (UFMS), Av. Senador Filinto Müller 2443, Campo Grande, MS 79074-460, Brasil.

${ }^{3}$ Faculdade de Medicina Veterinária e Zootecnia (FAMEZ), UFMS, Av. Senador Filinto Müller 2443, Campo Grande, MS 79074-460. *Autor para correspondência: ricardo.lemos@ufms.br

${ }^{4}$ Médico Veterinário autônomo, Veredas Pecuária de Corte, Rua 24 de outubro 674, Vila Carvalho, Campo Grande, MS 79004-400.

${ }^{5}$ Laboratório de Botânica, UFMS, Campo Grande, MS.

${ }^{6}$ Departamento de Patologia, Universidade Federal de Santa Maria (UFSM), Camobi, Santa Maria, RS 97105-900, Brasil.
}

piquete infestado pela planta por 37 dias. Os sinais clínicos consistiram em relutância em movimentar-se, andar trôpego (incoordenação), decúbito esternal permanente, diminuição do tônus de língua, estado comportamental em alerta, mioglobinúria caracterizada por urina castanho-escuro e fezes ressecadas com ou sem muco (ocasionalmente diarreicas com estrias de sangue). As principais alterações macroscópicas observadas nos 12 bovinos necropsiados estavam nos músculos esqueléticos dos membros pélvicos e foram caracterizadas por graus variáveis de palidez nos grupos musculares. Histologicamente, a lesão mais relevante encontrada foi degeneração e necrose segmentar multifocal nos músculos estriados esqueléticos (miopatia degenerativa tóxica multifocal polifásica). 0 diagnóstico da intoxicação baseou-se na epidemiologia (massa de forragem e de planta tóxica, análise da lotação do piquete e análise da precipitação pluviométrica), no quadro clínico dos animais e nos achados de necropsia e histopatologia. 
TERMOS DE INDEXAÇÃO: Plantas tóxicas, doenças de bovinos, Senna obtusifolia, Fabaceae, Caesalpinioideae, fedegoso, intoxicação por planta, Centro-Oeste, doença dos músculos estriados.

\section{INTRODUÇÃO}

Duas espécies de plantas do gênero Senna, S. occidentalis (Pierce \& O’Hara 1967, Barros et al. 1990, 1999, Carmo et al. 2011) e S. obtusifolia (Froehlich 2010, Queiroz et al. 2012), são descritas como tóxicas para bovinos provocando necrose muscular. A maioria dos surtos é causada pela ingestão de Senna occidentalis e a intoxicação natural por esta planta foi descrita em outras espécies animais como suínos domésticos (Martins et al. 1986), javalis (Sant'Ana et al. 2011) e provavelmente equinos (Barros et al. 1990). As intoxicações por $S$. occidentalis ocorrem tanto em bovinos criados em sistema extensivo por ingestão natural, quanto acidentalmente em animais que recebem suplemento concentrado contaminado com suas sementes (Barth et al. 1994, Barros et al. 1990, 1999, Carmo et al. 2011). Em um levantamento de 16 surtos ocorridos no estado do Rio Grande do Sul em bovinos em pastejo, Carmo et al. (2011) constataram que a doença tem sazonalidade definida de abril a julho (outono e inverno) com a maioria dos casos ocorrendo no mês de maio, em geral após a primeira geada ou após os primeiros dias frios. Além disso, os mesmos autores verificaram que a intoxicação por $S$. occidentalis é uma importante causa de morte em bovinos neste estado e que a mortalidade pode ser tão elevada quanto 55\%.

A intoxicação por Senna obtusifolia foi descrita em bovinos no Brasil nos estados de Santa Catarina e Paraná. A planta é considerada pouco palatável e o primeiro surto da intoxicação, ocorrido em duas propriedades, foi devido à ingestão de folhas e vagens verdes pelos bovinos em pastejo (Froehlich 2010). 0 segundo surto foi relatado em bovinos confinados que também ingeriram folhas e vagens da planta tóxica (Queiroz et al. 2012). Nos dois relatos, os coeficientes de morbidade foram $38 \%$, 4,5\% e $10 \%$, e de letalidade $87,5 \%, 100 \%$ e $95 \%$ respectivamente. Em surtos descritos em outros países, as condições que levaram à ingestão da planta foram a contaminação da ração com folhas, caules e sementes (McCormack \& Neisler 1980) ou somente folhas (Nicholson et al. 1977). Os coeficientes de morbidade e letalidade no surto relatado por McCormack \& Neisler (1980) foram 7\% e 100\%, respectivamente.

S. obtusifolia é uma planta da família Leg. Caesalpinoideae, comumente encontrada no Brasil, onde é conhecida como fedegoso, fedegoso-branco, mata-pasto e mata-pasto liso (Lorenzi 1991). Segundo Costa et al. (2002), é uma planta de 1,5-2.0m de altura, de crescimento ereto e caule sem espinhos. As folhas são paripinadas com três pares de folíolos, sem pelos e não quebradiças. As flores são em cacho com pétalas amarelas. As vagens são compridas, finas e curvas, com aproximadamente $10-12 \mathrm{~cm}$ de comprimento. A produção de sementes é elevada e as vagens quando "estalam", espalham as sementes que germinam no início das chuvas. Acredita-se que seus princípios tóxicos sejam um alcaloide, uma albumina tóxica, uma hepatotoxina, antraquinonas e possivelmente outros componentes (McCormack \& Neisler 1980).

Os principais sinais clínicos que os bovinos apresentam na intoxicação por plantas do gênero Senna são fraqueza muscular, incoordenação, tremores musculares, normorexia, estado comportamental em alerta, decúbito esternal permanente, diarreia, mioglobinúria e morte, sendo as lesões macroscópicas e microscópicas encontradas principalmente nos músculos esqueléticos, respectivamente como: áreas pálidas intercaladas com áreas aparentemente normais e miopatia tóxica degenerativa (McCormack \& Neisler 1980, Barros et al. 1990, 1999, Raffi et al. 2003, Carmo et al. 2011, Takeuti et al. 2011, Froehlich 2010, Queiroz et al. 2012).

Embora existam relatos de intoxicação por S. obtusifolia em bovinos no Brasil, o objetivo deste trabalho foi descrever os aspectos epidemiológicos, clínicos e patológicos observados em um surto de intoxicação por S. obtusifolia em bovinos em regime de pastejo, no Pantanal Sul-mato-grossense, caracterizado por elevada morbidade, enfatizando as condições que levaram os bovinos ao consumo da planta.

\section{MATERIAL E MÉTODOS}

O surto de intoxicação ocorreu em uma propriedade rural localizada no município de Miranda (20¹0'43,3587"S, 56³3'26,2921" 0), situada ao sul da região Centro-Oeste, no Pantanal Sul-mato-grossense. Na pastagem em que ocorreram as mortes havia Senna obtusifolia e Axonopus purpusii (forrageira nativa conhecida como capim-mimoso). Na pastagem havia 313 novilhas Nelore com idade de 24 meses. Os dados epidemiológicos e clínicos foram obtidos durante visitas à propriedade, realizadas durante todo o surto. Realizou-se colheita de exemplares da planta suspeita para identificação e classificação no Laboratório de Botânica e posteriormente para ser mantida no Herbário (CGMS- 37872), ambos da Universidade Federal de Mato Grosso do Sul (UFMS).

Para estimar a média do total de massa verde por hectare, tanto da forrageira como da planta tóxica, utilizou-se o método do quadrado, no qual se joga um molde de $0,5 \times 0,5 \mathrm{~m}\left(0,25 \mathrm{~m}^{2}\right) \mathrm{em}$ áreas representativas da pastagem a ser analisada e coletam-se amostras ao nível do solo de todas as plantas presentes no interior do molde metálico, realizando a pesagem logo em seguida. No presente caso, foram realizadas estimativas na pastagem onde ocorreram as mortes (P1) e para fins de estudo comparativo, o mesmo procedimento foi realizado na pastagem ao lado (P2) que também era composta pela mesma forrageira e continha S. obtusifolia. Na pastagem 2 não ocorrem casos da intoxicação.

Realizou-se um levantamento do índice pluviométrico da propriedade referente aos últimos cinco anos (2008-2013). Os dados foram registrados pe lo proprietário e coletados em uma das visitas à propriedade.

0 exame físico dos bovinos foi realizado segundo Dirksen et al. (1993). Procedeu-se eutanásia das novilhas tanto em fase inicial como em fase terminal para análise macro e microscópica. Foram colhidos, para exame histopatológico, fragmentos dos seguintes músculos: psoas, longíssimo dorsal, glúteo, semimembranoso, semitendinoso, vasto lateral, reto femoral, intercostal, diafragmático, deltoide e bíceps, além de miocárdio, fígado, rim, pulmão, linfonodos, baço, esôfago, língua e sistema nervoso central. Os fragmentos dos tecidos foram fixados em formol tamponado a $10 \%$, processados rotineiramente para histopatologia e corados pela hematoxilina-eosina (HE).

\section{RESULTADOS}

Os primeiros casos clínicos (16 animais) foram observados no dia 13 de fevereiro de 2013, nesta data, os bovinos foram retirados da P1 e, no trajeto para outra pastagem, mais 12 
animais manifestaram decúbito esternal permanente após a movimentação. A pastagem em que ocorreram as intoxicações (P1) possui área de 59,2 hectares e, no momento do surto, havia 313 novilhas que permaneceram neste local por 37 dias. Após a retirada dos bovinos da pastagem onde havia a planta, continuaram ocorrendo casos por mais 10 dias, totalizando 165 bovinos (morbidade 52,7\%). Todos os animais que adoeceram apresentaram evolução fatal (letalidade 100\%).

À inspeção da P1 (Fig.1), constatou-se grande quantidade de Senna obtusifolia com sinais de consumo pelos bovinos (presença de talos cortados com ausência de folhas na extremidade) (Fig.2). Em entrevistas com o médico veterinário responsável pela propriedade, o mesmo informou que a P1 fora utilizada em anos anteriores, porém em épocas diferentes e com menor carga animal, pois, durante o mês de fevereiro, esta área sempre fica alagada devido aos altos índices pluviométricos, impossibilitando a ocupação pelos bovinos neste piquete nesta época do ano. No ano em questão, como as chuvas foram escassas, não houve alagamento

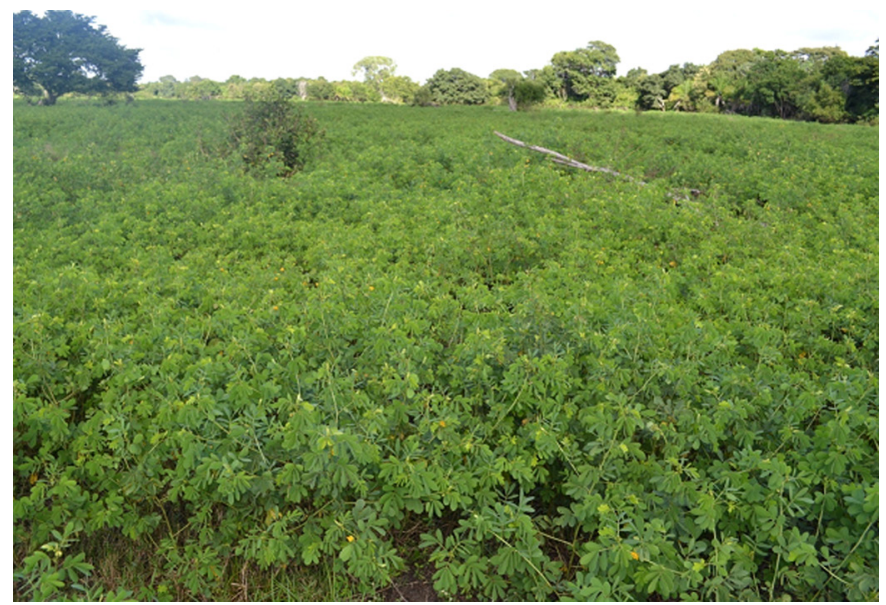

Fig.1. Intoxicação espontânea por Senna obtusifolia em bovinos no pantanal Sul-Mato-Grossense. Pastagem (P1) severamente invadida por S. obtusifolia.

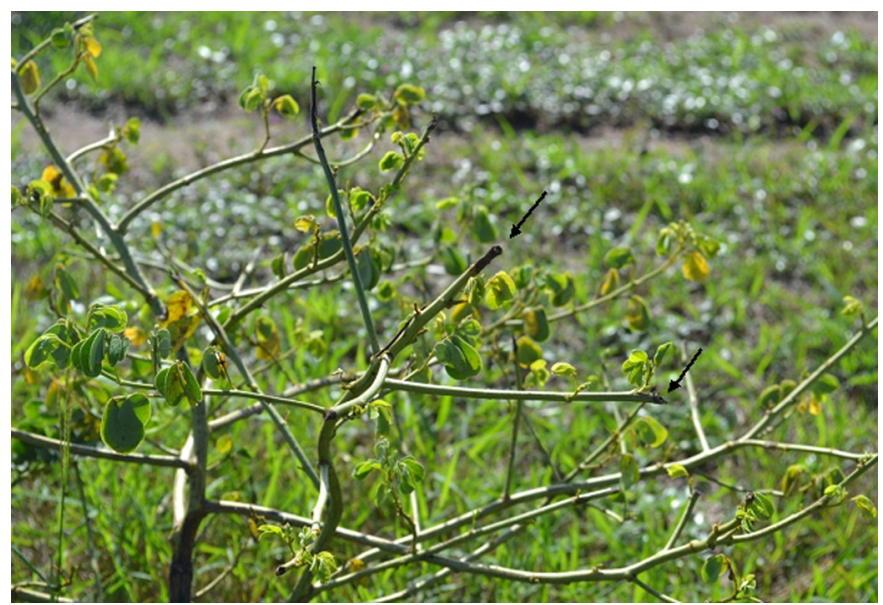

Fig.2. Intoxicação espontânea por Senna obtusifolia em bovinos no pantanal Sul-Mato-Grossense. Exemplares da planta com sinais de consumo pelos bovinos: talos cortados com ausência de folhas nas extremidades (setas). da área, possibilitando que as novilhas fossem colocadas nessa pastagem para maior aproveitamento da forragem e serem abatidas posteriormente. Os índices pluviométricos da propriedade encontram-se explicitados na Fig.3.

Na pastagem ao lado (P2), na qual havia presença de $S$. obtusifolia, porém não ocorreram casos de intoxicação, a área é de 202 hectares. A planta invasora se distribuía, de maneira não uniforme, por toda área das duas pastagens. Os principais dados epidemiológicos referentes ao surto encontram-se sumarizados no Quadro 1.

Foram examinadas sete novilhas, das quais quatro receberam tratamento à base de vitamina E e Selênio (40mL/ animal/dia ${ }^{7}$, porém sem resposta a esse tratamento. A doença foi caracterizada por evolução clínica que variou de 24 horas a 15 dias e sinais clínicos de fraqueza muscular, decúbito esternal e/ou lateral permanente (Fig.4), relutância em movimentar-se, estado comportamental em alerta,

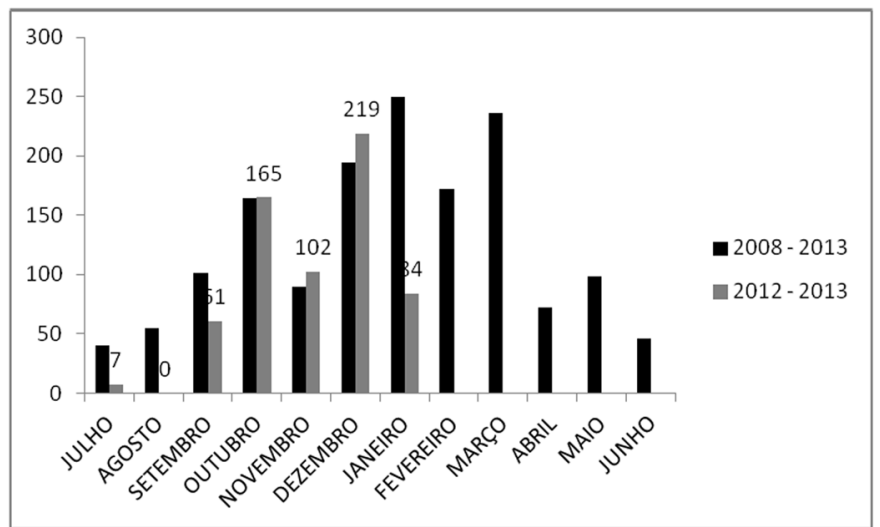

Fig.3. Intoxicação espontânea por Senna obtusifolia em bovinos no pantanal Sul-Mato-Grossense. Precipitação pluviométrica da fazenda na qual ocorreu o surto. Média dos últimos cinco anos (2008-2013) e precipitação no ano de 2012 a 2013.

Quadro 1. Dados epidemiológicos referentes ao surto de intoxicação espontânea por Senna obtusifolia em bovinos em pastagem

\begin{tabular}{lcc}
\hline $\begin{array}{l}\text { Variáveis } \\
\text { Categoria }\end{array}$ & $\begin{array}{c}\text { Pastagem 1 (P1) } \\
\text { Novilhas }\end{array}$ & $\begin{array}{c}\text { Pastagem 2 (P2) } \\
\text { Vacas e Touros }\end{array}$ \\
\hline Área (ha) & 59,2 & 202 \\
Número Animais & 313 & 300 \\
Lotação (U.A./ha) $_{\text {Fedegoso (kg MV/ha) }}^{\mathrm{c}}$ & $3,17^{\mathrm{a}}$ & $1,5^{\mathrm{b}}$ \\
Capim Mimoso (kg MV/hac $^{\mathrm{c}}$ & $11.755,0$ & $5.112,0$ \\
Capim Mimoso (kg MS/ha) $^{\mathrm{d}}$ & $3.255,8$ & $3.869,6$ \\
Demanda (kg MS/ha) $_{\text {Coeficiente de morbidade (\%) }}$ & 813,95 & 967,4 \\
Coeficiente de letalidade (\%) $^{2}$ & $100,8^{\mathrm{e}}$ & 52,7 \\
& 0 & $1.328,2^{\mathrm{f}}$
\end{tabular}

a (313 novilhas x $270 \mathrm{~kg}) / 450 \mathrm{~kg}=187,8 \mathrm{UA} / 59,2 \mathrm{ha}=3,17 \mathrm{UA} / \mathrm{ha} \cdot{ }^{\mathrm{b}}[(286$ vacas x $450 \mathrm{~kg}$ x $1 \mathrm{UA})+(14$ touros $\times 450 \mathrm{~kg} \times 1,3 \mathrm{UA})] / 450 \mathrm{~kg}=304,2$ $\mathrm{UA} / 202$ ha $=1,5 \mathrm{UA} /$ ha. $^{\mathrm{c}}$ Estimativa usando um quadrado metálico de $0,25 \mathrm{~m}^{2}$. ${ }^{\mathrm{d}}$ Estimativa considerando $25 \%$ de matéria seca. ${ }^{\mathrm{e}}(270 \mathrm{~kg} \mathrm{x} 4 \%$ de oferta x 37 dias de ocupação x 313 novilhas)/59,2 ha $=2.112,8 \mathrm{~kg}$ MS/ha. ${ }^{f}[(450 \mathrm{~kg} \times 4 \%$ de oferta $\times 49$ dias de ocupação $\times 286$ vacas $)+$ (585 $\mathrm{kg}$ x $4 \%$ oferta $\times 49$ dias $\times 14$ touros)] $/ 202$ ha = 1.328,2 $\mathrm{kg}$ MS $/ \mathrm{ha}$.

ESE Liquid $^{\circledR}$, Vetnil Ind. e Com. de Produtos Veterinários Ltda, Avenida José Nicolau Stabile 53. Burck, Louveira, SP 13290-000, Brasil. Site: www.vetnil.com.br 


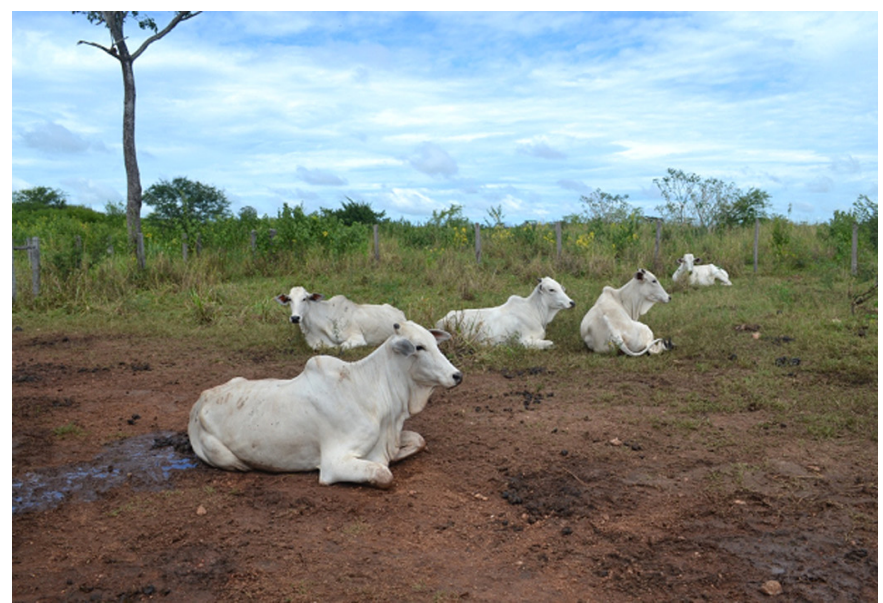

Fig. 4. Intoxicação espontânea por Senna obtusifolia em bovinos no pantanal Sul-Mato-Grossense. Novilhas intoxicadas pela planta manifestando decúbito esternal permanente e estado comportamental alerta.

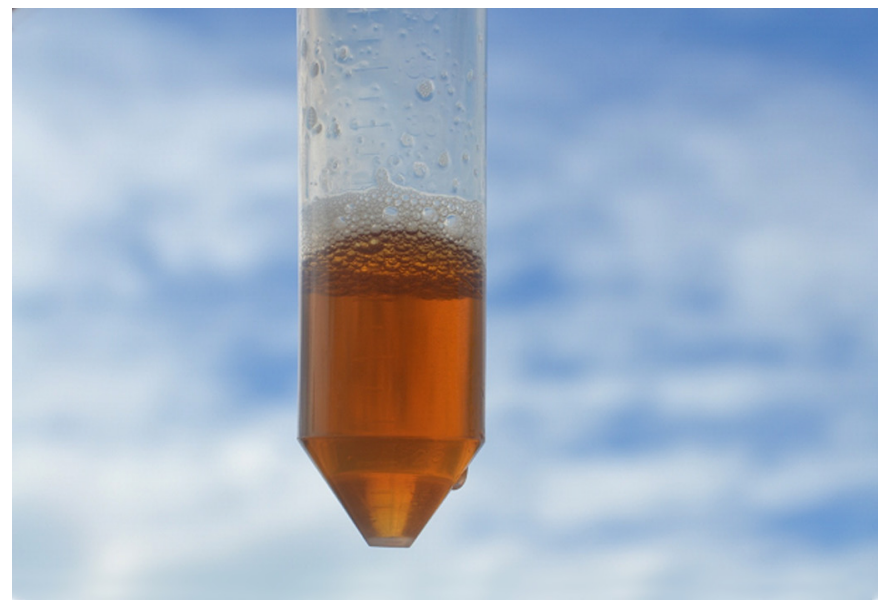

Fig.5. Intoxicação espontânea por Senna obtusifolia em bovinos no pantanal Sul-Mato-Grossense. Urina castanho-escura coletada de uma novilha intoxicada pela planta durante a necropsia.

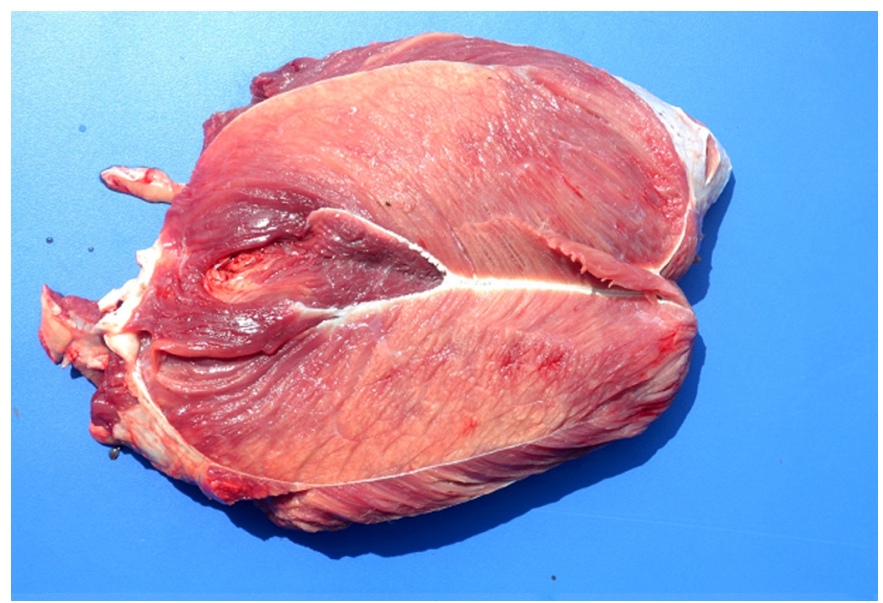

Fig.6. Intoxicação espontânea por Senna obtusifolia em bovinos no pantanal Sul-Mato-Grossense. Músculo quadríceps evidenciando áreas pálidas intercaladas com áreas de coloração normal.

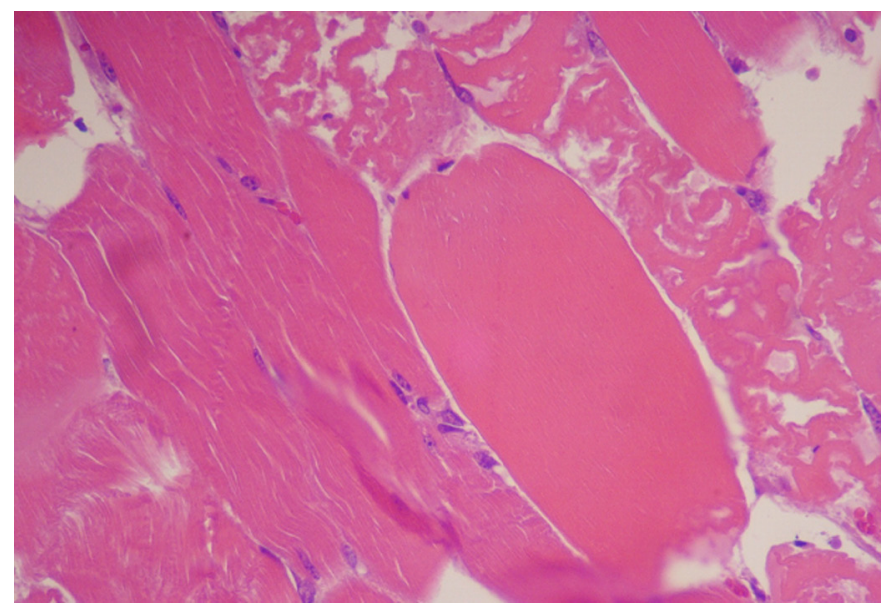

Fig.7. Intoxicação espontânea por Senna obtusifolia em bovinos no pantanal Sul-Mato-Grossense. Degeneração hialina e necrose flocular segmentar da musculatura esquelética. HE, obj.40x.

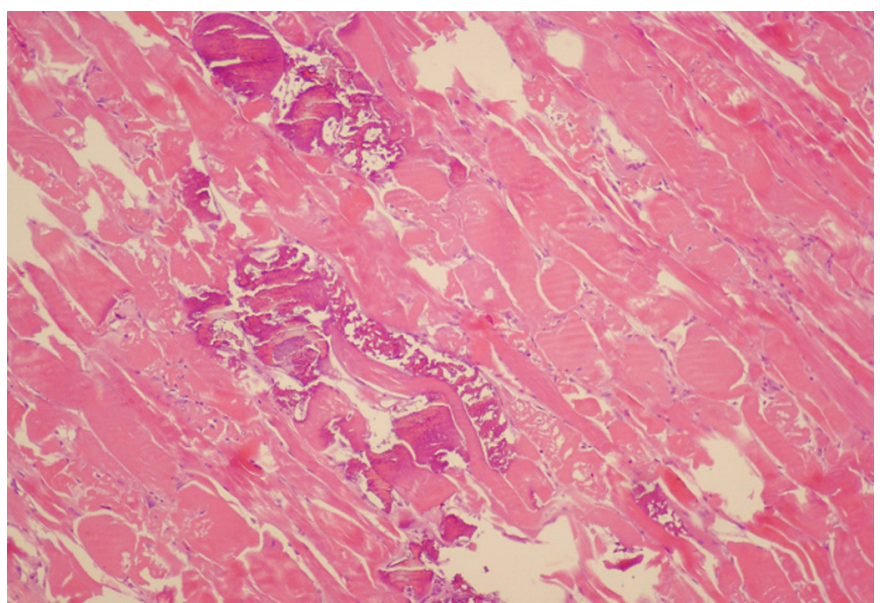

Fig.8. Intoxicação espontânea por Senna obtusifolia em bovinos no pantanal Sul-Mato-Grossense. Músculo esquelético, degeneração hialina, necrose e fragmentação de miofibras com mineralização. HE, obj.20x.

tônus de língua diminuído, fezes ressecadas com ou sem muco (ocasionalmente diarreicas com estrias de sangue), desidratação moderada, enoftalmia moderada, hipomotilidade ou atonia ruminal, taquicardia e taquipneia.

Realizou-se necropsia de 12 bovinos, duas de forma sistematizada (na qual há coleta identificada dos músculos). Os achados foram semelhantes nos animais avaliados. As lesões macroscópicas mais relevantes foram áreas pálidas assimétricas nos músculos esqueléticos, em forma de manchas alternadas com áreas de coloração normal ou listras claras (Fig.5). Essas áreas de palidez foram encontradas em todos os músculos coletados, porém com maior intensidade nas grandes massas musculares dos membros pélvicos. Mioglobinúria foi observada em nove dos 12 animais necropsiados (Fig.6).

As lesões histológicas observadas foram semelhantes em todos os bovinos, predominando o quadro de miopatia degenerativa segmentar multifocal. Essa lesão foi caracterizada por graus variáveis de degeneração hialina, necrose flocular (Fig.7), degeneração discoide, presença de infiltra- 


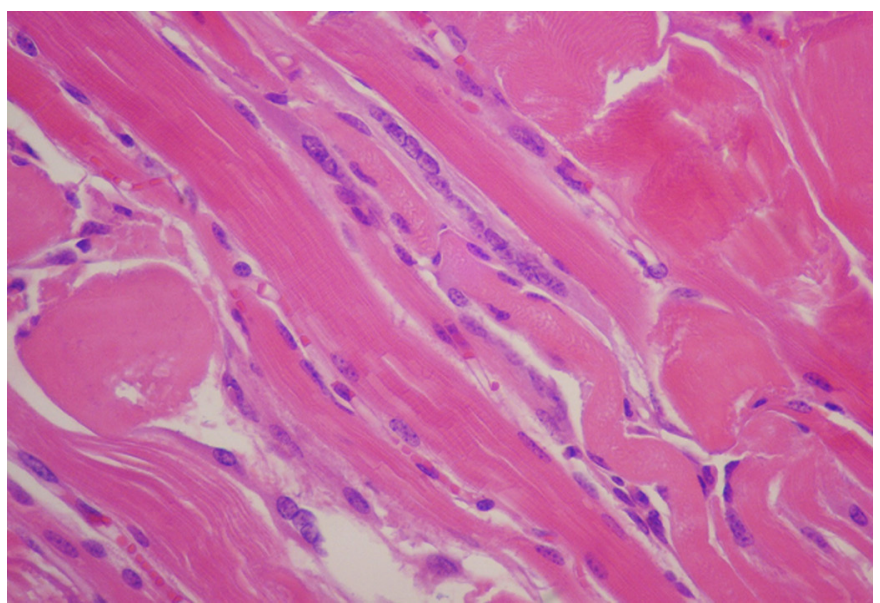

Fig. 9. Intoxicação espontânea por Senna obtusifolia em bovinos no pantanal Sul-Mato-Grossense. Músculo esquelético com alteração regenerativa caracterizada por formação de miotubos. HE, obj. 40x.

do mononuclear, edema intersticial, mineralização (Fig.8) e regeneração das miofibras com formação dos miotubos (Fig.9). Nos bovinos em fase inicial da intoxicação, não foram observadas mineralização e regeneração das fibras musculares esqueléticas. Na língua de cinco bovinos havia degeneração hialina, degeneração discoide e necrose flocular. Nos demais órgãos não houve alterações significativas.

\section{DISCUSSÃO}

O diagnóstico baseou-se nos achados epidemiológicos (grande quantidade da planta na pastagem e evidências de ingestão da mesma pelos bovinos), no quadro clínico e patológico, que foram semelhantes aos relatos anteriores de intoxicação por Senna obtusifolia (Froehlich 2010, Queiroz et al. 2012), assim como nos relatos de intoxicação por $S$. occidentalis, em que os achados são idênticos aos encontrados no surto em questão (Barros et al. 1990, 1999, Raffi et al. 2003, Carmo et al. 2011, Takeuti et al. 2011).

Os coeficientes de morbidade e letalidade $(52,7 \%$ e $100 \%$ ) observados nesse surto foram superiores a todos os demais relatos de intoxicação por S. obtusifolia. Os maiores valores relatados anteriormente para a intoxicação natural por esta planta em bovinos em pastejo foram respectivamente de 38\% e 100\% (Froehlich 2010), e para bovinos em confinamento de $10 \%$ e $95 \%$ (Queiroz et al. 2012). Como pode-se observar, o coeficiente de morbidade do presente surto é superior ao maior coeficiente descrito anteriormente. 0 coeficiente de letalidade do presente caso é o mesmo relatado por Froehlich (2010) em um dos surtos. Riet-Correa \& Méndez (2007) afirmaram que a intoxicação por $S$. occidentalis é frequentemente fatal, com letalidade próxima a $100 \%$. 0 mesmo deve ser considerado para intoxicação por S. obtusifolia, com base no coeficiente descrito em relatos anteriores (McCormack \& Neisler 1980, Froehlich 2010, Queiroz et al. 2012).

Nos quatro bovinos em que foi realizado tratamento com vitamina E e selênio, não houve resposta de melhora do quadro clínico. O'Hara et al. (1970) avaliaram os efeitos da vitamina E/selênio no tratamento de bovinos intoxica- dos experimentalmente por $S$. occidentalis e concluíram que a administração de vitamina E/selênio potencializou os efeitos de miotoxicidade da Senna, e que a vitamina E foi o componente mais importante nesta potencialização. No presente caso, não é possível atribuir esses efeitos ao tratamento, mas apenas concluir que o mesmo não foi eficaz.

Os fatores predisponentes para a ocorrência desse surto foram a alta taxa de lotação da P1, a grande quantidade de massa de $S$. obtusifolia e a escassez de forragem devido à falta de chuvas, levando a fome e consequente ingestão da planta tóxica pelos animais. Através do cálculo da estimativa de massa de forragem e da planta tóxica, associado à análise pluviométrica da propriedade, os fatores predisponentes foram determinados de forma precisa. Em surtos anteriores, tais fatores foram relatados de forma empírica. Sugere-se que esses métodos científicos sejam utilizados em trabalhos posteriores para maior acuráciadas informações obtidas.

O curso clínico da doença variou de 24 horas a 15 dias. Froehlich (2010) também encontrou casos de evolução aguda com morte em dois dias, e um caso em que o bovino permaneceu em decúbito por treze dias. No surto relatado por Queiroz et al. (2012), os animais apresentaram evolução clínica de cinco a nove dias. Nas intoxicações por S. occidentalis descritas por Carmo et al. (2011) o curso clínico também variou de dois a 15 dias.

Os principais sinais clínicos observados neste surto foram fraqueza muscular, relutância em se movimentar, decúbito esternal e/ou lateral permanente e fezes ressecadas. Achados semelhantes foram descritos em outras intoxicações por S. obtusifolia (Nicholson et al. 1977, McCormack \& Neisler 1980, Froehlich 2010, Queiroz et al. 2012) e em intoxicações por S. occidentalis (Barros et al 1990, Barth et al. 1994, Barros et al. 1999, Raffi et al. 2003). Destaca-se que seis bovinos examinados apresentaram diminuição do tônus de língua, porém todos mantiveram a movimentação da cauda, sendo este um aspecto importante no diferencial para o botulismo.

As lesões macroscópicas mais relevantes foram caracterizadas por áreas assimétricas de palidez na musculatura esquelética, principalmente dos membros pélvicos, alternadas com áreas aparentemente normais. Histologicamente, essa lesão caracterizou-se por graus variáveis de degeneração hialina, necrose flocular com infiltrado mononuclear e edema intersticial. Esses achados, macro e microscópicos, corroboram com relatos anteriores da intoxicação por Senna spp (Nicholson et al. 1977, McCormack \& Neisler 1980, Barros et al. 1990, 1999, Raffi et al. 2003, Froehlich 2010, Carmo et al. 2011, Takeuti et al. 2011, Queiroz et al. 2012). Alterações como degeneração discoide (Barros et al. 1990) e formação de miotubos (Barros et al. 1990, 1999, Carmo et al. 2011) não foram relatadas por Froehlich (2010) e Queiroz et al. (2012), porém no presente surto foram descritas, além da mineralização das miofibras (Barth et al. 1994, Froehlich 2010).

Mioglobinúria foi observada em 75\% dos animais necropsiados. Queiroz et al. (2012) relataram essa alteração em $37,5 \%$ dos animais avaliados clinicamente, já Froehlich (2010) não observou mioglobinúria, ao exame físico, em ne- 
nhum bovino. Esta alteração, caracterizada por urina marrom-avermelhada, é observada em $50 \%$ a $100 \%$ dos casos e é resultante da destruição da fibra muscular, subsequente liberação de mioglobina na circulação sanguínea e sua eliminação na urina (Pierce \& O'Hara 1967, Schmitz \& Denton 1977, Barros 1993). Em relatos anteriores de intoxicação por S. obtusifolia, Queiroz et al. (2012) verificaram necrose paracentral multifocal em três vacas e Froehlich (2010) observou necrose de hepatócitos na região centrolobular em um bovino intoxicado experimentalmente por folhas e vagens $(10 \mathrm{~g} / \mathrm{kg}$ peso vivo/seis dias). Este efeito hepatotóxico também foi descrito em intoxicações por $S$. occidentalis (Henson \& Dollahite 1966, Mercer et al. 1967, Rogers et al. 1979, Barros et al. 1990, Takeuti et al. 2011). Carmo et al. (2011), em estudo realizado a partir de surtos ocorridos no Rio Grande do Sul, sugerem que as lesões hepáticas encontradas não estariam correlacionadas com as lesões cardíacas descritas. O'Hara et al. (1969) consideraram que a lesão hepática nas intoxicações por $S$. occidentalis eram secundárias à insuficiência cardíaca congestiva, decorrente de lesão degenerativa no miocárdio. Lesões degenerativas no miocárdio foram descritas em intoxicações espontâneas por S. obtusifolia e S. occidentalis (Barros et al. 1999, Carmo et al. 2011, Queiroz et al. 2012) e experimentais (Henson \& Dollahite 1966, Mercer et al. 1967, Barros et al. 1990) com intensidades diferentes. No presente estudo não foram observadas alterações hepáticas e em miocárdio.

No diagnóstico diferencial devem ser incluídas outras doenças que causam necrose muscular, como intoxicação por S. occidentalis, deficiência de vitamina E/selênio e intoxicação por antibióticos ionóforos. Além disso, afecções que cursem com paresia, incoordenação e diminuição do tônus da língua devem ser descartadas, como é o caso do botulismo (Tokarnia et al. 2012).

\section{CONCLUSÃO}

Conclui-se que a associação de fatores como: uma categoria animal com maior exigência nutricional (novilhas), maior taxa de lotação da pastagem P1 e consequentemente maior demanda de forragem, juntamente com baixa oferta de capim-mimoso nesta pastagem com grande massa de Senna obtusifolia, levou as novilhas a restrição alimentar e a ingestão da planta tóxica com aparecimentos do quadro clínico característico da intoxicação.

Agradecimentos. -Ao Conselho Nacional de Desenvolvimento Científico e Tecnológico (CNPq no 14/2011 - Projeto Universal Proc.483211/2012-5).

\section{REFERÊNCIAS}

Barros C.S.L. 1993. Intoxicação por plantas que afetam o sistema muscular: intoxicação por Senna occidentalis, p.201-213. In: Riet-Correa F., Méndez M.C., Schild A.L. (Eds), Intoxicações por Plantas e Micotoxicoses em Animais Domésticos. Hemisferio Sur, Montevideo.

Barros C.S.L., Pilati C., Andujar M.B., Graça D.L., Irigoyen L.F., Lopes S.T. \& Santos C.F. 1990. Intoxicação por Cassia occidentalis (Leg. Caes.) em bovinos. Pesq. Vet. Bras. 10:47-58.

Barros C.S.L., Ilha M.R.S., Bezerra Jr P.S., Langohr I.M. \& Kommers G.D. 1999. Intoxicação por Senna occidentalis (Leg. Caesalpinoideae) em bovinos em pastoreio. Pesq. Vet. Bras. 19:68-70.
Barth A.T., Kommers G.D., Salles M.S., Wouters F. \& Barros C.S.L. 1994. Coffee senna (Senna occidentalis) poisoning in cattle. Vet. Human Toxicol. 36:541-545.

Carmo P.M.S., Irigoyen L.F., Lucena R.B., Fighera R.A., Kommers G.D. \& Barros C.S.L. 2011. Spontaneous coffee senna poisoning in cattle: report on 16 outbreaks. Pesq. Vet. Bras. 31(2):139-146

Costa J.A.S, Nunes T.S. \& Ferreira A.P.L. 2002. Leguminosas forrageiras da Caatinga; espécies importantes para as comunidades rurais do sertão da Bahia. Universidade Estadual de Feira de Santana. SASOP, Feira de Santana, BA. 132p.

Dirksen G., Gründer H.D. \& Stöber M. 1993. Rosenberger - Exame Clínico dos Bovinos. $3^{a}$ ed. Guanabara Koogan, Rio de Janeiro. 419p.

Froehlich D.L. 2010. Intoxicação espontânea e experimental por folhas e vagens da planta Senna obtusifolia (Leguminosae) em bovinos. Dissertação de Mestrado, Centro de Ciências Agroveterinárias, UDESC, Lages/ SC 38p.

Henson J.B. \& Dollahite J.W. 1966. Toxic myodegeneration in calves produced by experimental Cassia occidentalis intoxication. Am. J. Vet. Res. 27(119):947-949.

Lorenzi H. 1991. Plantas Daninhas do Brasil: terrestres, aquáticas, parasitas, tóxicas e medicinais. $2^{\mathrm{a}}$ ed. Plantarum, Nova Odessa. 268p.

Martins E., Martins V.M.V., Riet-Correa F., Soncini R.A. \& Paraboni S.V. 1986. Intoxicação por Cassia occidentalis (Leguminoseae) em suínos. Pesq. Vet. Bras. 6:35-38.

McCormack E. \& Neisler W.E. 1980. Cassia obtusifolia (sicklepod) toxicity in a dairy herd. Vet. Med., Small Anim. Clin. 75:1849-1851.

Mercer H.D., Neal F.C., Himes J.A. \& Edds G.T. 1967. Cassia occidentalis toxicosis in cattle. J. Am. Vet. Med. Assoc. 151(6):735-741.

Nicholson S.S., Thornton J.T. \& Rimes A.J. 1977.Toxic myopathy in dairy cattle caused by Cassia obtusifolia in greenchop. Bovine Pract. 12:120.

O'Hara P.J., Pierce K.R. \& Read W.K. 1969. Degenerative myopathy associated with ingestion of Cassia occidentalis L. Clinical and pathologic features of the experimentally induced disease. Am. J. Vet. Res. 30(12):21732180.

O’Hara P.J., Pierce K.R. \& Read K. 1970. Effects of vitamin E and Selenium on Cassia occidentalis intoxication in cattle. Am. J. Vet. Res. 31(12):21512156.

Pierce K.R. \& O’Hara P.J. 1967. Toxic myopathy in Texas cattle. South Western Veterinarian 20(2):179-184.

Queiroz G.R., Ribeiro R.C.L., Romão F.T.N.M.A., Flaiban K.K.M.C., Bracarense A.P.F.R.L. \& Lisbôa J.A.N. 2012. Intoxicação espontânea de bovinos por Senna obtusifolia no estado do Paraná. Pesq. Vet. Bras. 32(12):12631271.

Raffi M.B., Sallis E.S.V., Rech R.R.R., Garmatz S.L. \& Barros C.S.L. 2003. Intoxicação por Senna occidentalis em bovinos em pastoreio: relato de caso. Revta Fac. Zootec. Vet. Agron., Uruguaiana, 10(1):131-136.

Riet-Correa F. \& Méndez M.C. 2007. Intoxicações por plantas e micotoxinas, p.99-219. In: Riet-Correa F., Schild A.L., Lemos R.A.A. \& Borges J.R.J. (Eds), Doenças de Ruminantes e Equídeos. Vol.2. 3a ed. Pallotti, Santa Maria.

Rogers R.J., Gibson J. \& Reichmann K.G. 1979.The toxicity of Cassia occidentalis for cattle. Aust. Vet. J. 55(9):408-412.

Sant'Ana F.J.F., Garcia E.C., Rabelo R.E., Ferreira Júnior C.S., Freitas Neto A.P. \& Verdejo A.C.F. 2011. Intoxicação espontânea por Senna occidentalis em javalis (Sus scrofaferus) no Estado de Goiás. Pesq. Vet. Bras. 31(8):702706.

Schmitz D.G. \& Denton J.H. 1977. Senna bean toxicity in cattle. South Western Veterinarian 30(2):165-170.

Takeuti K.L., Raymundo D.L., Bandarra P.M., Oliveira L.G.S., Boabaid F.M., Barreto L. \& Driemeier D. 2011. Surto de intoxicação por Senna occidentalis em bovinos em pastoreio. Acta Scient. Vet. 39(1):954.

Tokarnia C.H., Brito M.F., Barbosa J.D., Peixoto P.V. \& Döbereiner J. 2012. Plantas Tóxicas do Brasil para Animais de Produção. 2ª ed. Helianthus, Rio de Janeiro. 586p. 